\title{
Inversion of Abelian Integrals on Small Genus Curves
}

Kevin R. Coombes and Robert J. Fisher, Jr.

Department of Mathematics, University of Michigan, Ann Arbor, MI 48109, USA

Department of Mathematics, University of Oklahoma, 601 Elm Avenue, Norman, OK 73019, USA

Let $M$ be a smooth projective curve of genus $g>0$. Let $J$ be the Jacobian of $M$ and let $M^{(i)}$ be the $i^{\text {th }}$ symmetric product. Fix a base point $P \in M$ and define a map $\phi_{i}: M^{(i)} \rightarrow J$ by $\phi_{i}(D)=D-i P$. Mattuck [6,7] has shown that, if $i \geqq 2 g-1$, then $\phi_{i}$ is a $\mathbb{P}^{i-g}$-bundle. The most interesting case occurs when $i=2 g-1$, since in [1] the bundles for larger $i$ are determined by this one.

The inversion of abelian integrals problem asks: What is an explicit description of the transition functions of the bundle $\phi_{i}$ ? For an excellent introduction to the problem, see Kempf's article [5]. Until now, the only complete answer was given in genus 1 by the Abel Inversion Theorem, the Riemann-Roch Theorem, and Riemann's approach through theta functions. Even without the transition functions, Gunning $[1,2]$ and Kempf $[3,4]$ were able to extract a great deal of information about $M$ from the bundles $\phi_{i}$.

In this paper, we present a solution to the inversion of abelian integrals problem in the cases

(i) curves of genus 2

(ii) non-hyperelliptic curves of genus 3 .

The techniques we use are very geometric but essentially elementary. We strongly emphasize the role played by effective divisors and by the Riemann-Roch Theorem. To build sections, we use the commutative diagram

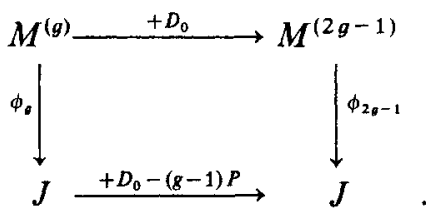

where $D_{0}$ is a fixed effective divisor of degree $g-1$. The Jacobi Inversion Theorem says that $\phi_{g}$ is a birational surjection; hence, it has an inverse on an open set. It is then necessary to choose enough different $D_{0}$ 's to trivialize the bundle. The geometry first enters when we must describe the open set over which a given collection of $D_{0}$ 's determines a trivialization. We then use explicit knowledge of the 
canonical embedding of $M$ to describe the transition functions. For precise statements, see Theorems $B$ and $C$,

Remarks. (i) Based on our results, it seems reasonable to expect that a trivializing atlas of $\phi_{2 g-1}: M^{(2 g-1)} \rightarrow J$ can be obtained using $g+1$ Zariski open sets, each of which has complement equal to a union of translates of the theta divisor. It should be of particular interest to interpret the geometry of such an explicit trivialization in terms of theta functions.

(ii) All our results apply equally well to the linearized inversion of abelian integrals problem [5]. It is only necessary to supply the correct technical means for translating the section $D_{0}+\phi_{g}^{-1}(\quad)$ into a section of the linearized bundle.

(iii) The critical geometric fact in the genus 3 case is the isomorphism between the space $\Gamma(M, \mathscr{L}(2 K))$ of quadratic differentials and the space $\Gamma\left(\mathbb{P}^{2}, \mathcal{O}(2)\right]$ of conics. For curves of higher genus, there is merely a surjection

$$
\Gamma\left(\mathbb{P}^{g-1}, \mathcal{O}(2)\right) \rightarrow \Gamma(M, \mathscr{L}(2 K)) .
$$

More care will therefore be needed to exploit this geometry. It is intriguing to note that

$$
\begin{aligned}
& \operatorname{dim} \Gamma\left(\mathbb{P}^{g-1}, \mathcal{O}(2)\right)=\frac{g^{2}+g}{2}=\operatorname{dim} \mathscr{A}_{g} \\
& \operatorname{dim} \Gamma(M, \mathscr{L}(2 K))=3 g-3=\operatorname{dim} \mathscr{M}_{g}
\end{aligned}
$$

where $\mathscr{A}_{g}$ is the moduli space of principally polarized abelian varieties of dimension $g$ and $\mathscr{M}_{g}$ is the moduli space of curves of genus $g$. One might hope that an explicit description from this point of view would provide further insight into the Schottky problem.

\section{Geometric Preliminaries}

We begin with a brief review of the problem, emphasizing divisors rather than line bundles. Let $M$ be a smooth projective curve of positive genus. A divisor on $M$ is a finite collection $D=\Sigma n_{i} P_{i}$ of points $P_{i} \in M$ with multiplicities $n_{i} \in \mathbb{Z}$. The degree of $D$ is $\operatorname{deg}(D)=\Sigma n_{i}$. If all $n_{i} \geqq 0$, then $D$ is called effective.

To each divisor $D$ is associated a locally free sheaf $\mathscr{L}(D)$ of rank one on $M$. On small open sets $\mathscr{U}_{i}, D \cap \mathscr{U}_{i}$ is the divisor of zeros and poles of some rational function $f_{i}$ on $\mathscr{U}_{i}$. The transition functions of $\mathscr{L}(D)$ are given by multiplication by $f_{i} / f_{j}$, which is invertible on $\mathscr{U}_{i} \cap \mathscr{U}_{j}$. We will write $\ell(D)=\operatorname{dim} \Gamma(M, \mathscr{L}(D))$.

Two divisors are called linearly equivalent, written $D \sim D^{\prime}$, provided there exists a rational function $f$ on $M$ with divisor $(f)=D-D^{\prime}$. If $s \in \Gamma(M, \mathscr{L}(D))$, then its divisor of zeros $(s)_{0}$ determines $s$ up to a constant. So, the projectivization $\mathbb{P}(D):=\mathbb{P}(\Gamma(M, \mathscr{L}(D)))$ is the space of effective divisors on $M$ which are linearly equivalent to $D$.

Let $J$ denote the Jacobian of $M$, which parametrizes linear equivalence classes of divisors of degree zero. Let $M^{(i)}$ be the $i^{\text {th }}$ symmetric product. A point $D \in M^{(i)}$ is an effective divisor of degree $i$. Choose a base point $P \in M$ and define

$$
\phi_{i}: M^{(i)} \rightarrow J \quad \phi_{i}(D)=D-i P .
$$


Moreover, let $W_{i}$ denote $\phi_{i}\left(M^{(i)}\right)$ in $J$. Mattuck [6] showed that, if $i \geqq 2 g-1$, then $\phi_{i}$ is a projective bundle whose fiber over $R \in J$ is $\mathbb{P}^{i-g}=\mathbb{P}(R+i P)$. The problem of inverting abelian integrals on $M$ asks:

What is an explicit description of the transition functions of $\phi_{i}$ ?

Gunning [1, Theorem 11] reduces the problem to the case $i=2 g-1$. From now on, we restrict our attention to this case.

We approach the problem from a very geometric point of view. Firstly, recall the following fundamental facts.

Theorem (Riemann-Roch). Let $K$ be the canonical divisor on $M$. Let $D$ be any divisor. Then

$$
\ell(D)=\ell(K-D)+\operatorname{deg}(D)+1-g .
$$

Theorem (Jacobi Inversion). The map $\phi_{g}: M^{(g)} \rightarrow J$ is a surjective birational equivalence.

If $\operatorname{deg}(D) \geqq 2 g-1$, then $\ell(K-D)=0$. The Riemann-Roch Theorem gives a computation of $\ell(D)$ that depends only on the degree; this is the first indication that $\phi_{i}$ should be a bundle. If $0<\operatorname{deg}(D)<2 g-1$ and $\ell(K-D)>0$, then we say that $D$ is special. Most divisors are non-special; the Jacobi Inversion Theorem reflects this fact for divisors of degree $g$. Write $B \subset J$ for the closed set on whose complement $\phi_{g}$ is an isomorphism.

We now describe our basic technique to get a section of $\phi_{2 g-1}$ over a comprehensible open subset of $J$.

Proposition 1. Fix an effective divisor $D_{0}$ on $M$ of degree $g-1$. Let $B_{0}$ be the translate in $J$ of $B$ by $D_{0}-(g-1) P$. Then $D_{0}$ determines a section

$$
\sigma_{0}: J-B_{0} \rightarrow M^{(2 g-1)}
$$

of $\phi_{2 g-1}$, which is characterized by the fact that the divisor $\sigma_{0}(R)-D_{0}$ is effective. Proof. There is a commutative diagram

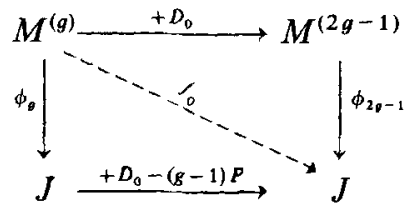

The composite $f_{0}$, by the Jacobi Inversion Theorem, has an inverse on $J-B_{0}$. Take

$$
\sigma_{0}(R)=f_{0}^{-1}(R)+D_{0} .
$$

It is clear that $\sigma_{0}(\mathrm{R})-D_{0}$ is effective of degree $g$, and must be the unique effective representative of its divisor class when $R \in J-B_{0}$.

An explicit description of the transition function still requires two steps. Firstly, describe how to choose a collection $D_{0}^{(1)}, \ldots, D_{0}^{(g+1)}$ of effective divisors of degree $g-1$ in such a way that the corresponding sections trivialize $M^{(2 g-1)}$ on a describable open set. Secondly, give an explicit description of how the sections change when the collection of effective divisors is changed. 
We illustrate the usefulness of emphasizing divisors with the following result, which we will need later. Define $f: M \rightarrow J$ by $f(X)=X+K-(2 g-1) P$. Notice that $f$ is the translation of the usual embedding $\phi_{1}$ by the degree zero divisor class $K-(2 g-2) P$; that is, $f(M)=W_{1}+K-(2 g-2) P$.

Theorem A. The pullback $f^{*}\left(\phi_{2 g-1}\right)$ is a trivial bundle on $M$, with fibre naturally isomorphic to $\mathbb{P}(K)$.

Proof. Let $X \in M$. The fibre over $X$ is given by projectivizing

$$
\Gamma(M, \mathscr{L}(f(X)+(2 g-1) P))=\Gamma(M, \mathscr{L}(K+X)) .
$$

But $\ell(K+X)=\ell(K)=g$ and there is a natural inclusion

$$
\Gamma(M, \mathscr{L}(K)) \subset \Gamma(M, \mathscr{L}(K+X)) \text {. }
$$

By dimension counting, this inclusion is an isomorphism. The fibre is therefore independent of the point $X$ and the pullback bundle is trivial.

\section{Curves of Genus Two}

Let $M$ be a curve of genus 2 . In this section we study the $\mathbb{P}^{1}$-bundle $\phi_{3}: M^{(3)} \rightarrow J$. As in Proposition 1, the choice of a single point $Q_{0} \in M$ determines a section $\sigma_{0}$ of $\phi_{3}$, which is characterized by the fact that

$$
\sigma_{0}(R)=E_{0}(R)+Q_{0}
$$

where $E_{0}(R)$ is the unique effective divisor linearly equivalent to $R+3 P-Q_{0}$. The section is defined on an open set $J-B_{0}$ for a translate $B_{0}$ of $B$, the image under $\phi_{2}$ of the locus of special divisors of degree 2 .

Lemma 2. $B_{0}$ is a single point of $J$; namely, $B_{0}=\left[K+Q_{0}-3 P\right]$.

Proof. The canonical class is the unique special divisor class of degree 2 on the hyperelliptic curve $M$.

Now let $Q=\left\{Q_{0}, Q_{1}, Q_{\infty}\right\}$ be an ordered triple of points of $M$ which lie in distinct fibres of the canonical hyperelliptic map of $M$ to $\mathbb{P}^{1}$, and which are also not ramification points of this map. There are three corresponding sections of $\phi_{3}$ on some open set of $J$. A trivialization of $\phi_{3}$ is determined over the open set where these sections take on distinct values.

Proposition 3. Let $\mathscr{U}$ be the maximal open set where the sections corresponding to $Q$ trivialize $\phi_{3}$. Then $J-\mathscr{U}$ consists of the union of three translates of the theta divisor; namely,

$$
\begin{aligned}
& \mathrm{W}_{1}+\left[Q_{0}+Q_{1}-2 P\right] \\
& W_{1}+\left[Q_{0}+Q_{\infty}-2 P\right] \\
& W_{1}+\left[Q_{1}+Q_{\infty}-2 P\right]
\end{aligned}
$$

Proof. By symmetry, it is enough to determine the locus where $\sigma_{0}(R)=\sigma_{1}(R)$. These sections are equal if and only if there is an equality of divisors

$$
E_{0}(R)+Q_{0}=E_{1}(R)+Q_{1} .
$$


Since $Q_{0} \neq Q_{1}$, the latter equality implies

$$
\begin{aligned}
& E_{0}(R)=Q_{1}+X \\
& E_{1}(R)=Q_{0}+X
\end{aligned}
$$

for some $X \in M$. Hence the dependency locus is

$$
\left\{R \in J: R \sim Q_{0}+Q_{1}+X-3 P \quad \text { for some } \quad X \in M\right\}=W_{1}+\left[Q_{0}+Q_{1}-2 P\right] \text {. }
$$

Proposition 4. Let $Q$ and $\bar{Q}$ be two triples of points which determine trivializations of $\phi_{3}$ on open sets $\mathscr{U}$ and $\overline{\mathscr{U}}$. Assume all 6 points of $Q \cup \bar{Q}$ have canonically distinct images. Then $J-(\mathscr{U} \cup \overline{\mathscr{U}})$ consists of exactly 18 points.

Proof. This is a special case of the Poincare relation. It suffices to check that

$$
I=W_{1}+\left[Q_{0}+Q_{1}-2 P\right] \cap W_{1}+\left[\bar{Q}_{0}+\bar{Q}_{1}-2 P\right]
$$

contains exactly 2 points. If $R \in I$, then there exist $X, Y \in M$ such that

$$
R+3 P \sim X+Q_{0}+Q_{1} \sim Y+\bar{Q}_{0}+\bar{Q}_{1} .
$$

Write ${ }^{*}$ for the hyperelliptic involution on $M$. Then

$$
\begin{aligned}
X+Y^{*} & \sim\left(Y+\bar{Q}_{0}+\bar{Q}_{1}\right)-\left(Q_{0}+Q_{1}\right)+Y^{*} \\
& \sim K+\left(\bar{Q}_{0}+\bar{Q}_{1}-Q_{0}-Q_{1}\right) .
\end{aligned}
$$

By the assumption on $Q$ and $\bar{Q}$, the latter is a nontrivial translate of the canonical class, hence nonspecial. So, it has a unique effective representative $S+T$. Thus, the only 2 points of $I$ occur when

$$
\begin{array}{cl}
\text { (i) } X=S & Y=T^{*} \\
\text { (ii) } X=T & Y=S^{*} .
\end{array}
$$

Proposition 5. There exist 3 triples $Q, \bar{Q}, \overline{\bar{Q}}$ such that the union of the corresponding trivializing neighborhoods is

$$
\mathscr{\mathscr { U }} \cup \overline{\mathscr{U}} \cup \overline{\overline{\mathscr{U}}}=J .
$$

Proof. Let $Q$ and $\bar{Q}$ be any 2 triples as in Proposition 4. It suffices to choose $\bar{Q}$ so that the translates of the theta divisor by pairs of points in $\overline{\bar{Q}}$ miss the 18 points of the complement of $\mathscr{U} \cup \overline{\mathscr{U}}$. This is an open condition.

Finally, we address the question of how to describe the transition functions. Fix a model of $M$ of the form

$$
M: y^{2}=h(x)
$$

where $h(x)$ is a monic polynomial of degree 6 with distinct zeroes. Fix a triple $Q$. A general point $R \in J$ determines a unique pair $X, Y \in M$ with

$$
X+Y \sim R+3 P-Q_{0} .
$$

Functions on $J$ will be described by symmetric functions in $X$ and $Y$.

Lemma 6. Suppose $X, Y, Q_{0}$ and $Q_{\infty}^{*}$ lie in distinct fibres of the canonical map. Then there is a unique cubic $C$ of the form

$$
C: y=f(x)
$$

such that $C$ passes through all 4 points. 
Proof. If all 4 points lie in the affine $(x, y)$-plane, the result is clear. The potential difficulties arise if one of the points lies over the singularity at infinity in the planar model. Homogenize the equations of $M$ and $C$ in the standard way and pass to the affine $y=1$ where

$$
\begin{aligned}
& M: z^{4}=\prod_{i=1}^{6}\left(x-a_{i} z\right) \\
& C: z^{2}=d \prod_{i=1}^{3}\left(x-b_{i} z\right) .
\end{aligned}
$$

The successive blow-ups which desingularize both curves can be summarized by the substitutions

$$
z=t u^{3} \quad x=t u^{2}
$$

whence

$$
\begin{aligned}
M: 1 & =t^{2} \prod\left(1-a_{i} u\right) \\
C: 1 & =d t \prod\left(1-b_{i} u\right) .
\end{aligned}
$$

The exceptional points over $\infty$ occur where $u=0$. Thus, $M$ meets $C$ at a point over $\infty$ if and only if $d= \pm 1$. Since this imposes exactly one condition on $C$, the result follows.

The curves $M$ and $C$ intersect in points which are determined by the zeroes of

$$
h(x)-f(x)^{2}=0 .
$$

This is a sextic equation, with four zeroes given by the $x$-coordinates of $X, Y, Q_{0}$, and $Q_{\infty}^{*}$. Factor these out and let $q(x)$ denote the monic residual quadratic. Define a rational function in $M$ by

$$
\gamma=\gamma_{Q, R}(x, y)=(y-f(x)) /\left(x-x_{\infty}\right) q(x),
$$

where $Q_{\infty}=\left(x_{\infty}, y_{\infty}\right)$. For fixed $Q$ and $R, \gamma_{Q, R}$ is a degree 3 map to $\mathbb{P}^{1}$ such that

$$
\begin{aligned}
\left(\gamma_{Q, R}\right)_{0} & =X+Y+Q_{0}=\sigma_{0}(R) \\
\left(\gamma_{Q, R}\right)_{\infty} & =\sigma_{\infty}(R) .
\end{aligned}
$$

In order to normalize this function correctly, let

$$
c_{Q, R}=\gamma_{Q, R}\left(Q_{1}\right) \text {. }
$$

If $R \in \mathscr{U}$ is a point where the hypotheses of Lemma 6 hold, then $c_{Q, R}$ is a non-zero constant. Define

$$
\psi_{Q, R}=\gamma_{Q, R} / c_{Q, R} .
$$

So, $\psi_{Q, R}$ determines an isomorphism

$$
\mathbb{P}(\Gamma(M, \mathscr{L}(R+3 P))) \approx \mathbb{P}^{1} .
$$

As $R$ varies, the trivialization of $\phi_{3}$ made explicit by $\psi$ agrees with the one defined abstractly as corresponding to $Q$.

Unfortunately, the definition of $\psi$ does not make sense for all $R \in \mathscr{U}$. The difficulty occurs when 2 of the 4 points $X, Y, Q_{0}, Q_{\infty}^{*}$ lie in the same canonical fibre. 
If 2 such points are equal, then there is no problem since one has merely specified the tangent direction to $C$ at a point. If $X=Y^{*}$, then the point

$$
R=\left[X+Y+Q_{0}-3 P\right]=\left[K+Q_{0}-3 P\right] \notin \mathscr{U},
$$

so there is again no problem. Finally, what happens when $X=Q_{0}^{*}$ ? Then $R+3 P \sim K+Y(R)$ for uniquely determined $Y(R)$ and the appropriate definitions are

$$
\begin{aligned}
& \gamma_{Q, R}=\left(x-x_{0}\right) /\left(x-x_{\infty}\right) \\
& \psi_{Q, R}=\gamma_{Q, R} / \gamma_{Q, R}\left(Q_{1}\right) .
\end{aligned}
$$

In any case, let $\psi_{Q, R}^{-1}(z)$ also denote the divisor naturally defined by the fibre of $\psi_{Q, R}$ at $z \in \mathbb{P}^{1}$. For most $R \in \mathscr{U}, \psi_{Q, R}^{-1}(z)$ has degree 3 and is linearly equivalent to $R+3 P$. When it does not, it has degree 2 ; and this case was described above. So explicitly a trivialization on $\mathscr{U}$ is given as follows:

$$
\psi^{-1}: \mathscr{U} \times P^{1} \rightarrow \phi_{3}^{-1}(\mathscr{U})
$$

is defined by

$$
\psi^{-1}(R, z)= \begin{cases}\psi_{Q, R}^{-1}(z) & \text { if } \psi \text { is a degree } 3 \text { map to } \mathbb{P}^{1} \\ \psi_{Q, R}^{-1}(z)+Y(R) & \text { otherwise. }\end{cases}
$$

One can check, essentially by l'Hôpital's Rule, that $\psi_{Q, R}^{-1}$ varies continuously with $R \in \mathscr{U}$. By construction, $\psi$ is the trivialization determined by $Q$.

Theorem B. Let $Q$ and $\bar{Q}$ be two triples of points of $M$ which determine trivializations of $\phi_{3}$ on open sets $\mathscr{U}$ and $\overline{\mathscr{U}}$. Then the transition function $\tau=\psi \circ \bar{\psi}^{-1}$ is completely determined by

$$
\begin{gathered}
\tau(0)=\psi\left(\bar{Q}_{0}\right) . \\
\tau(1)=\psi\left(\bar{Q}_{1}\right) . \\
\tau(\infty)=\psi\left(\bar{Q}_{\infty}\right) .
\end{gathered}
$$

Proof. The equations hold by construction. Since $\tau$ is an automorphism of a $\mathbb{P}^{1}$ bundle, it is determined by its values on the three points 0,1 , and $\infty$.

\section{Non-Hyperelliptic Curves of Genus Three}

Let $M$ be a non-hyperelliptic curve of genus 3 . The canonical model of $M$ is a degree 4 plane curve; a canonical divisor is a 4-tuple of collinear points. Fix a base point $P \in M$ to define the $\mathbb{P}^{2}$-bundle $\phi_{5}: M^{(5)} \rightarrow J$. In order to describe sections, we need to understand special divisors.

Lemma 7. Every special divisor of degree 3 on $M$ is linearly equivalent to $K-X$ for some $X \in M$.

Proof. If $D$ is special, then $\ell(K-D)>0$. Since $\operatorname{deg}(K-D)=1$ and $M$ is not a rational curve, an effective representative of $K-D$ is a uniquely determined point $X \in M$. Hence $D \sim K-X$. 
In the notation of Sect. 1, the lemma says that

$$
B=-W_{1}+[K-4 P] \text {. }
$$

Let $D_{0}$ be an effective divisor of degree 2 on $M$. By Proposition 1, $D_{0}$ determines a section

$$
\sigma_{0}(R)=D_{0}+E_{0}(R)
$$

where $E_{0}(R)$ is the unique effective representative of the class $R+5 P-D_{0}$. The section $\sigma_{0}$ is defined on the open set $J-B_{0}$ where

$$
B_{0}=B+\left[D_{0}-2 P\right]=-W_{1}+\left[K+D_{0}-6 P\right] .
$$

To trivialize $\phi_{5}$, it will be necessary to specify four independent sections. Let $Q=\left\{Q_{0}, \ldots, Q_{3}\right\}$ be an ordered 4-tuple of points of $M$, no 3 of which are collinear. View the subscripts as elements of the cyclic group $\mathbb{Z} / 4$. Now define four sections of $\phi_{5}$ by

$$
\sigma_{i}(R)=Q_{i}+Q_{i+1}+E_{i}(R) .
$$

Also, define $\Delta_{i}=W_{2}+\left[Q_{i}+Q_{i+1}+Q_{i+2}-3 P\right]$.

Proposition 8. Let $\mathscr{U}$ be the maximal open set in $J$ over which the collection of sections $\left\{\sigma_{i}\right\}$ trivializes $\phi_{5}$. Then

$$
J-\mathscr{U}=\bigcup_{i=0}^{3} \Delta_{i} .
$$

Proof. Fix $R \in J$. Write $S_{i}=\sigma_{i}(R) \in \mathbb{P}(R+5 P)$. If no 3 of the $S_{i}$ are collinear, then there is a unique isomorphism $F_{R}: \mathbb{P}(R+5 P) \rightarrow \mathbb{P}^{2}$ such that

$$
\begin{aligned}
& F_{R}\left(S_{0}\right)=(1: 0: 0) \\
& F_{R}\left(S_{1}\right)=(0: 1: 0) \\
& F_{R}\left(S_{2}\right)=(0: 0: 1) \\
& F_{R}\left(S_{3}\right)=(1: 1: 1) .
\end{aligned}
$$

So, it is enough to determine when 3 of the $S_{i}$ are collinear. By symmetry under the action of the cyclic group, it suffices to determine this for $S_{1}, S_{2}, S_{3}$.

If $S_{1}=S_{2}$, then there is an equality of divisors

$$
Q_{1}+Q_{2}+E_{1}(R)=Q_{2}+Q_{3}+E_{2}(R) \text {. }
$$

Cancel the common term $Q_{2}$. Since $Q_{1} \neq Q_{3}$, this equality forces $Q_{3}$ to lie in the support of $E_{1}(R)$. Therefore

$$
\mathrm{R} \sim Q_{1}+Q_{2}+E_{1}(R)-5 P \sim Q_{1}+Q_{2}+Q_{3}+X+Y-5 P
$$

for some $X, Y \in M$. So, $S_{1}=S_{2}$ if and only if $R \in \Delta_{1}$.

Suppose $S_{1} \neq S_{2}$. The line spanned by these two points in $\mathbb{P}(R+5 P)$ is just $L=\mathbb{P}\left(R+5 P-Q_{2}\right)$. So, $S_{3} \in L$ if and only if $S_{3}-Q_{2}$ is effective. Since the $Q_{i}$ are distinct, this can only occur if $E_{3}(R)-Q_{2}$ is effective. But then

$$
R \sim Q_{0}+Q_{3}+E_{3}(R)-5 P \sim Q_{0}+Q_{3}+Q_{2}+X+Y-5 P
$$

and $R \in \Delta_{2}$. So $S_{1}, S_{2}, S_{3}$ are collinear if and only if $R \in \Delta_{1} \cup \Delta_{2}$. The result now follows by symmetry. 
Proposition 9. Let $Q^{j}=\left\{Q_{0}^{j}, \ldots, Q_{3}^{j}\right\}$ be a 4-tuple on $M$ which determines a trivialization of $\phi_{5}$ on a maximal open set $\mathscr{U}^{j}$. A complete trivializing atlas for $\phi_{5}$ can be obtained by choosing 4 such general collections $Q^{j}$.

Proof. Choose $\mathrm{Q}^{1}$ arbitrarily. Define

$$
Y^{j}=J-\mathscr{U}^{1}-\ldots-\mathscr{U}^{j} .
$$

Assume inductively that the first $j-1$ collections have been chosen so that $Y^{j-1}$ has codimension $j-1$ in $J$. Write

$$
Y^{j-1}=\bigcup_{s} Z_{\mathrm{s}}
$$

as a union of irreducible components. For each $s$, pick a point $R_{s} \in Z_{s}$.

Choose $Q_{0}^{j}, Q_{1}^{j}$ to be points general enough and not lying in an earlier collection so that $R_{\mathrm{s}}+5 P-Q_{0}^{j}-Q_{1}^{j}$ has a unique effective representative, which is denoted $A_{s}$. Then $A=\bigcup_{s} \operatorname{supp}\left(A_{s}\right)$ is finite. Choose $Q_{2}^{j} \in M \backslash A$ general enough so that $R_{s}+5 P-Q_{i}^{j}-Q_{2}^{j}$ has a unique effective representative $B_{s}^{i}(i=0,1)$. Also let $B^{i}=\bigcup_{s} \operatorname{supp}\left(B_{s}^{i}\right)$ for $i=0,1$. Let $C=A \cup B^{0} \cup B^{1}$. Then $C$ is also finite. Choose $Q_{3}^{j} \in M-C$. By construction, $R_{s} \in \mathscr{U}^{j}$ for each $s$. Therefore,

$$
\operatorname{codim}\left(Y^{j}, J\right)=j \text {. }
$$

Since $J$ is 3-dimensional, $Y^{4}=\emptyset$.

In order to describe the transition functions, we must first develop a better geometric interpretation of the fibre $\mathbb{P}(R+5 P)$ of $\phi_{5}$ over $R \in J$. Let $D(R)$ be an effective representative of the class $2 K-R-5 P$. By Proposition $1, D(R)$ exists and is generically unique. Non-uniqueness occurs when $D(R)$ is special; i.e., when

$$
2 K-R-5 P \sim K-X \quad R \sim K+X-5 P .
$$

Let $M_{0}=W_{1}+[K-4 P], V_{0}=J-M_{0}$. So $D(R)$ is uniquely determined if and only if $R \in V_{0}$.

Proposition 10. If $R \in V_{0}$, then $\Gamma(M, \mathscr{L}(R+5 P))$ is naturally isomorphic to the vector space $\mathscr{C}(D(R))$ of all conics in $\mathbb{P}^{2}$ through the points $D(R)$.

Proof. As usual, multiple points of $D(R)$ should be thought of as infinitely near points corresponding to the tangent directions of $M$. Since $R \in V_{0}$, the points of $D(R)$ are non-collinear and the vector space $\mathscr{C}(D(R))$ has the correct dimension.

Since $M$ is canonically embedded, the space $\Gamma(M, \mathscr{L}(2 K))$ is naturally identified with the space of all conics in $\mathbb{P}^{2}$. Under this identification, the subspace $\mathscr{C}(D(R))$ of conics with specified base points $D(R)$ is the image of

$$
\Gamma(M, \mathscr{L}(R+5 P))=\Gamma(M, \mathscr{L}(2 K-D(R))) \subset \Gamma(M, \mathscr{L}(2 K)) .
$$

This interpretation can be extended over all of $J$. By Theorem A, $\phi_{5}$ restricts to the trivial bundle on $M$ with fibre $\mathbb{P}(K)=$ space of lines in $\mathbb{P}^{2}$. For $R \in M_{0}, D(R)$ consists of three points on a fixed line $L_{X}$ through $X \sim K-D(R)$. So, $\mathscr{C}(D(R))$ consists of the reducible conics $L \cdot L_{X}$ for varying $L$.

Corollary 11. The $\mathbb{P}^{2}$-bundle $\phi_{5}: M^{(5)} \rightarrow J$ is isomorphic to the bundle whose fibre over $R \in J$ is $P(\mathscr{C}(D(R)))$. 
Let $Q$ be a 4-tuple on $M$ defining a trivialization of $\phi_{5}$ on the open set $\mathscr{U}$. The conic $C_{i} \in \mathbb{P}(\mathscr{C}(D(R)))$ which corresponds to the section $\sigma_{i}(R)$ is the unique conic through the five points

$$
Q_{i}+Q_{i+1}+D(R)
$$

Pick arbitrary equations $\gamma_{i} \in \mathscr{C}(D(R))$ whose locus of zeroes is $C_{i}$. These equations need to be normalized correctly. For each $R \in \mathscr{U}$, we have

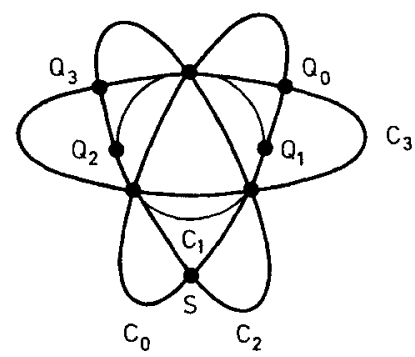

The common unlabelled intersection points form the support of $D(R)$. The point $S$ is defined to be the unique fourth intersection point of $C_{0}$ and $C_{2}$. Write $\gamma=\left(\gamma_{0}: \gamma_{1}: \gamma_{2}\right)$. Then $\gamma$ is a quadratic transformation of $\mathbb{P}^{2}$ which, when restricted, determines a morphism $\gamma_{M}: M \rightarrow \mathbb{P}^{2}$.

$$
\begin{aligned}
\gamma\left(Q_{1}\right) & =(0: 0: 1) \\
\gamma(S) & =(0: 1: 0) \\
\gamma\left(Q_{2}\right) & =(1: 0: 0) .
\end{aligned}
$$

Normalize the choice of the $\gamma_{i}$ so that

$$
\frac{\gamma_{1}}{\gamma_{2}}\left(Q_{0}\right)=-1 \quad \frac{\gamma_{0}}{\gamma_{1}}\left(Q_{3}\right)=-1
$$

Then the normalized $\gamma$ is completely determined by the fixed 4-tuple $Q$ and the variable $R \in \mathscr{U}$.

Proposition 12. Let $Q$ be a 4-tuple of points of $M$ determining a trivializing neighborhood $\mathscr{U}$ for $\phi_{5}$. Let $R \in \mathscr{U}$ and $F_{R}: \mathbb{P}(R+5 P) \rightarrow \mathbb{P}^{2}$ be the isomorphism described abstractly in Proposition 8. Then $F_{R}$ is induced by the quadratic transformation $\gamma$ given above.

Proof. Let $\pi: \widetilde{\mathbb{P}}^{2} \rightarrow \mathbb{P}^{2}$ be a minimal resolution of the indeterminacy of $\gamma$. So, $\pi$ is a regular birational map and there exists a regular map $\tilde{\gamma}: \widetilde{\mathbb{P}}^{2} \rightarrow \mathbb{P}^{2}$ yielding a commutative diagram

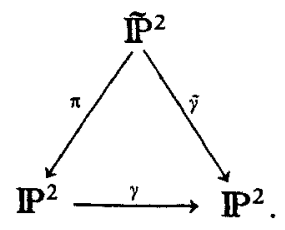


Let $E$ be the exceptional divisor in $\tilde{\mathbb{P}}^{2}$. For any divisor $D$ in $\mathbb{P}^{2}$, let $\tilde{D}$ denote its proper transform. Notice that

$$
\tilde{C}_{i} \cdot \tilde{M}=C_{i} \cdot M-D(R) .
$$

Take coordinates $\left(x_{0}: x_{1}: x_{2}\right)$ in $\mathbb{P}^{2}$. Let $H_{i}$ be the hyperplane $x_{i}=0$ and let $H$ be the hyperplane $x_{0}+x_{1}+x_{2}=0$. Then $\gamma^{*}\left(x_{i}\right)=\gamma_{i}$ for $i=0,1,2$. So

$$
\tilde{\gamma}^{*}\left(H_{i}\right)=\tilde{C}_{i} \text {. }
$$

Therefore

$$
\gamma_{M}^{*}\left(H_{i}\right)=\tilde{C}_{i} \cdot \tilde{M}=C_{i} \cdot M-D(R)=\sigma_{i}(R)
$$

for $i=0,1$, 2. In particular, $\gamma_{M}^{*} \mathcal{O}_{\mathbb{P}^{2}}(1)=\mathscr{L}(R+5 P)$ and hence $\gamma_{M}$ induces an isomorphism

$$
\left(\gamma_{M}^{*}\right)^{-1}: \mathbb{P}(R+5 P) \rightarrow \mathbb{P}^{2}=\mathbb{P}\left(\Gamma\left(\mathbb{P}^{2}, \mathcal{O}(1)\right)\right) .
$$

The previous computation shows that $F_{R}$ and $\left(\gamma_{M}^{*}\right)^{-1}$ agree on the three points $C_{0}, C_{1}, C_{2}$. But, because of the normalization,

$$
h=\gamma^{*}\left(x_{0}+x_{1}+x_{2}\right)=\gamma_{0}+\gamma_{1}+\gamma_{2}
$$

is such that $h\left(Q_{0}\right)=h\left(Q_{3}\right)=0$. Thus

$$
\hat{\gamma}^{*}(H)=\tilde{C}_{3}
$$

and

$$
\gamma_{M}^{*}(H)=\sigma_{3}(R)
$$

Therefore, $F_{R}=\left(\gamma_{M}^{*}\right)^{-1}$

Theorem C. Let $Q, \bar{Q}$ be two 4-tuples on $M$ which define trivializations of $\phi_{5}$ on $\mathscr{U}, \overline{\mathscr{U}}$, respectively. Let $\tau=F \circ \bar{F}^{-1}$ be the transition function. Then $\tau$ is completely determined by

$$
\begin{aligned}
\tau(0: 0: 1) & =\gamma\left(Q_{1}\right) \\
\tau(1: 0: 0) & =\gamma\left(\bar{Q}_{2}\right) \\
\tau(1:-1: 0) & =\gamma\left(\bar{Q}_{3}\right) \\
\tau(0: 1:-1) & =\gamma\left(\bar{Q}_{0}\right) .
\end{aligned}
$$

Proof. The equalities hold by the construction of $\gamma$ and by Proposition 12 . The 4 specified points of $\mathbb{P}^{2}$ are in general position. An automorphism of $\mathbb{P}^{2}$ is determined by its action on 4 points in general position.

\section{References}

1. Gunning, R.C.: Riemann surfaces and generalized theta functions. Berlin, Heidelberg, New York: Springer 1976

2. Gunning, R.C.: On generalized theta functions. Am. J. Math. 104, 183-208 (1982) 
3. Kempf, G.: Toward the inversion of abelian integrals. I. Ann. Math. 110, 243-273 (1979)

4. Kempf, G.: Toward the inversion of abelian integrals. II. Am. J. Math. 101, 184-202 (1979)

5. Kempf, G.: Inversion of abelian integrals. Bull. Am. Math. Soc. (New Ser.) 6, 25-32 (1982)

6. Mattuck, A.: Picard bundles. Ill. J. Math. 5, 550-564 (1961)

7. Mattuck, A.: Symmetric products and Jacobians. Am. J. Math. 83, 189-206 (1961)

8. Mumford, D.: Abelian varieties. Oxford: Oxford University Press 1970

Received July 4, 1985 